\title{
POTENCIAL DE MERCADO DE LA PAPA EN NICARAGUA
}

\section{Pedro Noel Torrez Rodríguez}

Investigador FDR-UNA, teléfono №. 22531971

E-mail: pedro0104_rodriguez@yahoo.es

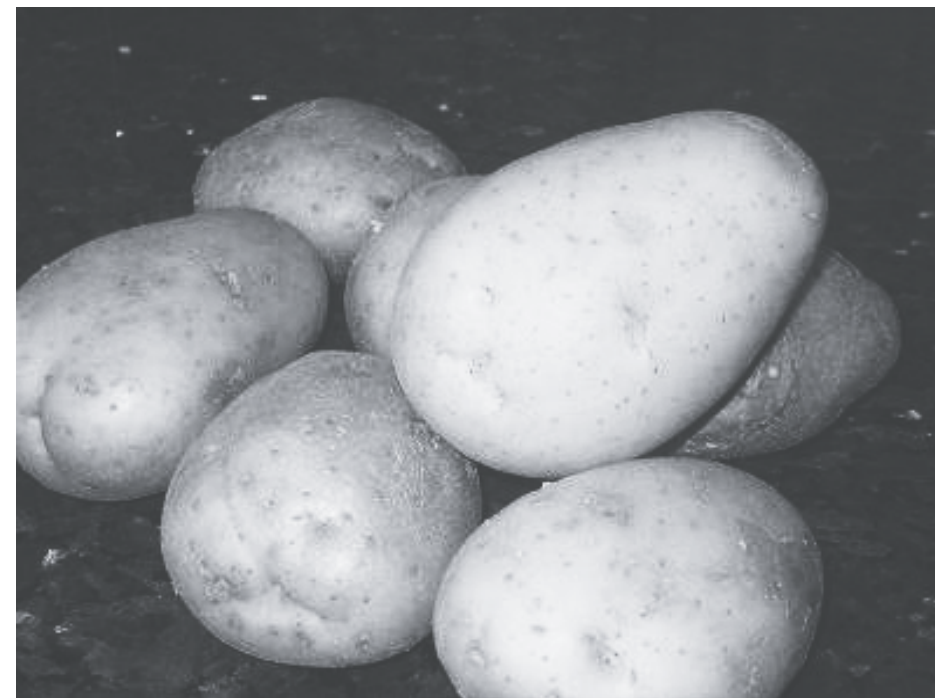

\section{RESUMEN}

El estudio fue realizado el mes de julio del 2008, con el propósito de analizar información clave de tipo comercial específicamente de ocho (8) mercados municipales del país; para que productores y demás interesados dispongan de información de mercado para la toma de decisiones económico-social en el rubro papa. Para el estudio se utilizó información escrita de fuentes del sector agrario, además de fuentes directas como informantes claves de ocho mercados municipales de un universo de 292 puestos de venta, de los cuales se seleccionaron 131 puestos como muestra de estudio. Las variables del estudio versaron sobre oferta y demanda, proyección y tipo de demanda, calidad y ventanas de mercado en diferentes periodos del año. Los resultados reflejan que la producción de papas frescas en Nicaragua es deficitaria en comparación a la demanda global, considerando un percapita de 8 kilogramos (17.6 libras) en el consumo anual. Esto significa que el país depende de las importaciones que oscilan en un 40\% y que provienen principalmente del área Centro Americana para satisfacer el déficit que cada año se manifiesta. Se proyectó la demanda insatisfecha a partir del año 2010 al 2020 considerando la producción local, el tipo de demanda, consumo percapita y viabilidad comercial; y los resultados marcan una tendencia decreciente respecto a las importaciones vía sustitución de importaciones con producción nacional. Las tendencias indican que dado la demanda y lo atractivo desde el punto de vista económico del rubro, los productores locales se ven estimulados en el futuro a incrementar el número de áreas y rendimientos por unidad producida,

\section{ABSTRACT}

The present study was carried out in July 2008, with the purpose to analyze potato marketing key data at country level. The study was carried out in eight municipal markets in order to produce information that could be available to farmers and others people who are interested in making social economic decisions concerning potato production. To carry out this study, written information from agricultural sources was used, as well as direct sources and key informants from eight municipal markets from a universe of 292 stalls, out of which 131 were selected as sample. The variables of the study focused on supply and demand, design and type of demand, quality and market windows at different times of the year. The results indicate that fresh potato production in Nicaragua is deficient compared to global demand, with a per-capita consumption of 8 kilograms (17.6 pounds) yearly. This means that the country relies on imports that range $40 \%$ mostly from Central American to meet the deficit that occurs every year. The unmet demand was projected starting from 2010 to 2018 considering the domestic production, the type of demand, per capita consumption, and marketing viability. The outcome shows a decreasing tendency with respect to importation by replacing importation by domestic production. This indicates that demand and the good prices that this products could get in the markets is attractive to farmers attractive from the economical point of view. The local farmers will be stimulated in the future to increase the area planted and the yield per unit area, reducing the need to import. Central America, Nicaragua is the country with the higher number of appropriate land to 
reduciendo la dependencia del exterior. Nicaragua es el país en Centro América con mayor cantidad de tierras aptas para la agricultura en diferentes rubros. Sin embargo, la producción de papas registra los menores rendimientos por área y países debido a la baja calidad de la semilla, inadecuados métodos y técnicas de cultivo, de control de plagas y enfermedades y manejo post cosecha. Las ventanas de mercado por escasez del producto ocurren en los meses de mayo a julio y de octubre a diciembre. Sobre las características de la papa nacional, los entrevistados resaltan la resistencia a la manipulación, el rendimiento y la duración o vida útil del producto. Entre los defectos mencionaron lo sucio, y pequeño del producto. La opinión sobre las características de la papa importada, está enfocada a la limpieza, buen tamaño y selección del producto. Entre los aspectos negativos de la papa importada se resalta la poca resistencia a la manipulación y sabor insípido del producto. Sobre la base de las características de la nacional y extranjera; el reto es producir una papa que reúna requisitos de resistencia, rendimiento, solides (nicaragüense) y buen tamaño (importada). En Nicaragua existen áreas agrícolas aptas para el rubro (Estelí, Matagalpa, Jinotega), poca competencia en la producción interna, rubro atractivo como negocio por su aceptable rentabilidad y una tendencias a incrementar el consumo percapita por su valor nutritivo e importancia en la seguridad alimentaria nacional.

Palabras clave: potencial de mercado, proyección de demanda, rendimientos de producción, ventanas de mercado y calidad del producto. plant different products. However, potato production recorded lower yield per unit area and countries due to low seed quality, inadequate farming methods and techniques, inadequate control of pests and diseases and post harvest management. The shortages windows market of potato occur from May to July and from October to December. About the qualities of the potato, the distributors stated that potatoes must be wholesome, clean, and big. And about the characteristics of the domestic potatoes, the survey highlighted resistance to manipulation, yield, and the long life of the product. Among the failing, they mentioned dirtiness, and small size of the product. The opinion about characteristics of the imported potatoes, it is focus on cleanliness, size, and selection of the product. There is a potential market because the demand of potatoes is not fulfilled. Nicaragua imports more than $40 \%$ of the potato that is consumed in Nicaragua. There is land apt for potato production (Estelí, Matagalpa, Jinotega), and little competition in the internal production. It is an attractive product for business because of its acceptability, profitability and there is a tendency to increase the consumption per capita due to its nutritional value and its importance in the national feeding safety.

Keywords: Potential potato market, demand and projection, yields, market window and quality of the product.

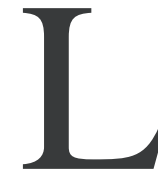

a papa es nativa del Perú, cuya antigüedad data de 7000 años antes de las culturas pre incas e incas. Es uno de los cultivos más importantes del mundo, para el consumo humano solamente es superado por tres cereales: el trigo, el arroz y el maíz (MAGFOR, 2008).

Es un alimento, muy nutritivo que desempeña funciones energéticas debido a su alto contenido en almidón así como funciones reguladoras del organismo por su elevado contenido en vitaminas hidrosolubles, minerales y fibra. Además, tiene un contenido no despreciable de proteínas, presentando un valor biológico relativamente alto dentro de los alimentos de origen vegetal.

En Nicaragua la producción de papas para consumo fresco es deficitaria, debiendo importar entre el 35 al 40\% para suplir la demanda nacional, debido a la escasez de semilla de calidad, altos costos de producción, manejo agronómico y acceso a fuentes de financiamiento (Comisión Nacional de papa Matagalpa, 2007).

Desde el punto de vista comercial existe desconocimiento del potencial y aprovechamiento del mercado de la papa, del valor nutritivo, del valor económico y de la importancia en la alimentación humana.
Con el presente estudio titulado potencial de mercado de la papa, se contempla analizar información clave de tipo comercial a nivel del país y específicamente de ocho mercados municipales; para que productores y demás interesados dispongan de información que permita la toma de decisiones económico-social sobre el rubro papa.

Las variables de estudio fueron oferta y demanda, proyección de la demanda insatisfecha, tipo de demanda, producción, calidad y meses de escasez del producto. Las fuentes de información utilizadas fueron informantes claves de los puestos de distribución de ocho mercados municipales, 131 puestos de distribución y de fuentes secundarias escritas proveniente de instituciones del gobierno como de organismos no gubernamentales que trabajan en este rubro.

El estudio provee información del potencial de mercado, proyección de la demanda, áreas de siembras versus sustitución de importaciones, tipo de demanda, requisitos de calidad y meses de escasez a través de todo el año. 


\section{MATERIALES Y MÉTODOS}

El estudio es de tipo descriptivo dado que identifica y caracteriza el universo de investigaciones, señala formas de conducta y establece relación entre variables. Para ello se utilizó el método deductivo partiendo de lo general a lo particular y haciendo uso de instrumentos como la entrevista y la encuesta (Méndez, 2005).

Se utilizaron datos estadísticos de producción y consumo nacional a partir del año 2001-2009 para relacionarlo con la demanda potencial en dichos años e inferir el consumo potencial proyectado a 10 años haciendo uso del método de regresión lineal. De igual manera se consideró información proveniente de centros de distribución comercial de mercados municipales para conocer la percepción con relación a los gustos y preferencias del producto tanto nacional como extranjero.

Para fuentes primarias se consideró un universo de 292 puestos de venta de ocho mercados municipales y una muestra razonada de 131 puestos de distribución.

\section{RESULTADOS Y DISCUSIÓN}

Si la demanda global es mayor a la oferta actual, se dice que hay demanda insatisfecha y que podría ser atractivo producir para satisfacer la demanda interna; en dependencia de la magnitud o tamaño del mercado. Un producto o servicio es viable comercialmente, cuando la demanda es suficientemente grande para decidir, producir y definir el grado de participación en dicha demanda. Si la demanda global es menor a la oferta actual, se dice que hay un exceso de producción y que no es atractivo entrar a producir en ese mercado (Torrez, 2009).

De acuerdo al balance oferta - demanda 2001-2009, la producción ofertada de papas para consumo fresco en Nicaragua fue menor a la demanda global, existiendo un déficit o demanda insatisfecha entre el 30 y el 36\% anual. El tamaño del mercado no cubierto es alto, que se corresponde a las importaciones que oscilan entre el 30 y 36\% de la demanda nacional provenientes del área Centro Americana.

Tabla 1. Variables, unidades de medida y conceptualización

\begin{tabular}{|c|c|c|}
\hline Variables & Medida & Indicador \\
\hline Oferta y demanda & qq & $\begin{array}{l}\text { Indicador que mide el grado de déficit o superhabit medido en quintales del } \\
\text { producto ofertado y demandado en ciertos periodos de tiempo. }\end{array}$ \\
\hline $\begin{array}{l}\text { Proyección de la } \\
\text { demanda }\end{array}$ & qq & $\begin{array}{l}\text { Indicador que mide la demanda proyectada en el tiempo, así como las áreas en } \\
\text { manzanas a ser sustituidas con producción nacional. }\end{array}$ \\
\hline Tipo de demanda & Alta, media, baja & $\begin{array}{l}\text { Mide la percepción de la demanda en los distribuidores mediante el } \\
\text { movimiento en las ventas medidas en demanda alta, media y baja. }\end{array}$ \\
\hline Rendimiento & $\mathrm{qq} / \mathrm{Mz}$ & $\begin{array}{l}\text { Indicador que mide los índices productivos por países en quintales por } \\
\text { manzana en los respectivos países de la región Centro Americana. }\end{array}$ \\
\hline Criterios de calidad & $\begin{array}{l}\text { Criterios propios de los } \\
\text { distribuidores }\end{array}$ & $\begin{array}{l}\text { Indicador que mide la percepción sobre los criterios de calidad del producto } \\
\text { (Sana, limpia, grande), características y ventajas de la papa nacional y de la } \\
\text { extranjera. }\end{array}$ \\
\hline $\begin{array}{l}\text { Meses de escasez } \\
\text { del producto }\end{array}$ & Meses & $\begin{array}{l}\text { Indicador que mide las ventanas de mercado que coinciden con los meses de } \\
\text { escasez del producto. }\end{array}$ \\
\hline
\end{tabular}

Diseñado el estudio se procedió a la búsqueda de información de fuentes secundarias, validación de instrumentos con usuarios directos, incorporación de sugerencias y finalmente visita a los puestos de venta ubicados en ocho mercados municipales. Se utilizó el programa SPSS para procesar la información, hacer los análisis correspondientes y redactar el informe de resultados.
En la proyección de la demanda a partir del año 2010, se observa un comportamiento decreciente del déficit; la que es sustituida con producción nacional (Figura 2).

La tendencia decreciente de las importaciones implica producir respectivamente entre 1000 manzanas en el 2010 a 350 manzanas en el año 2020, hasta reducir las importaciones vía sustitución con producción nacional. 


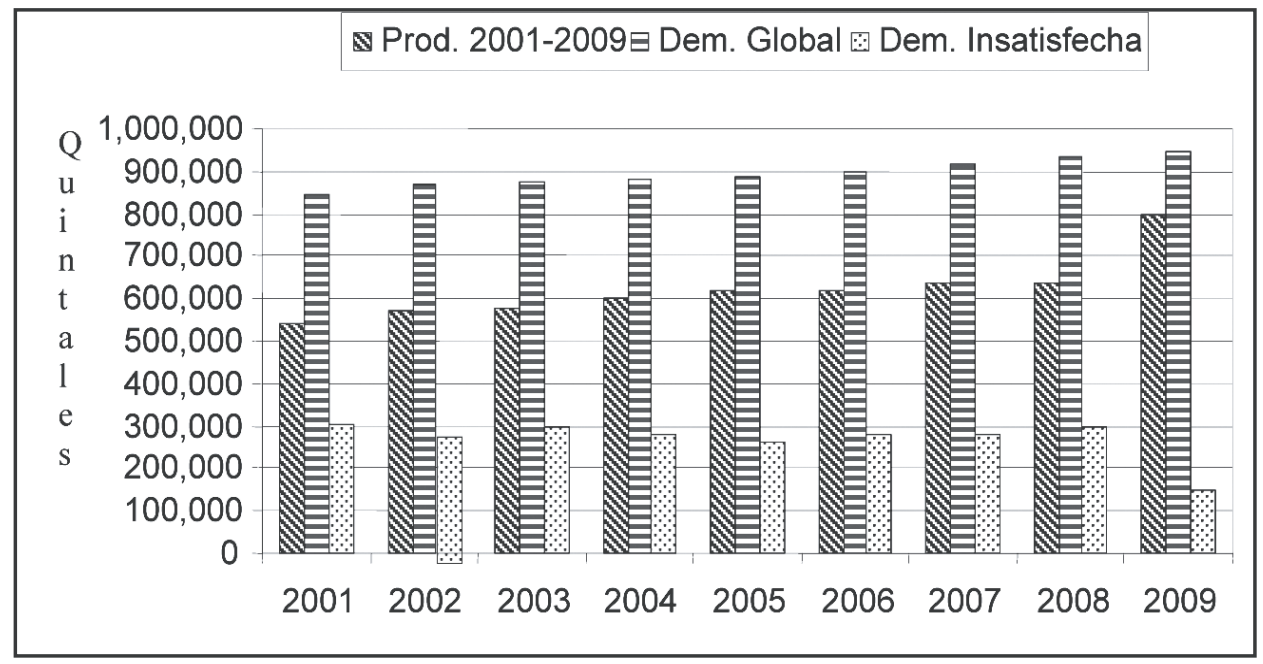

Figura 1. Balance producción - demanda de quintales de papa. Fuente: Adaptado de foasted (2005) y MAGFOAR (2008).

La sustitución de importaciones le ahorraría al país aproximado entre 3 y 4 millones de Dólares anuales. El incremento en la producción nacional debe estar acompañada de políticas de estímulos a la producción vía tecnificación, uso de semillas certificadas, precios atractivos y estables e incremento en el consumo percapita; para garantizar la estabilidad del rubro, la seguridad alimentaria y nutricional en la población nacional.
Nicaragua es el país en Centro América con mayor cantidad de tierras aptas para la agricultura en diferentes rubros. Sin embargo, la producción de papas registra los menores rendimientos por área en la región, debido a la baja calidad de la semilla, métodos y técnicas de cultivo, incidencia de plagas y enfermedades y manejo inadecuado de post cosecha (Foastad, 2005).

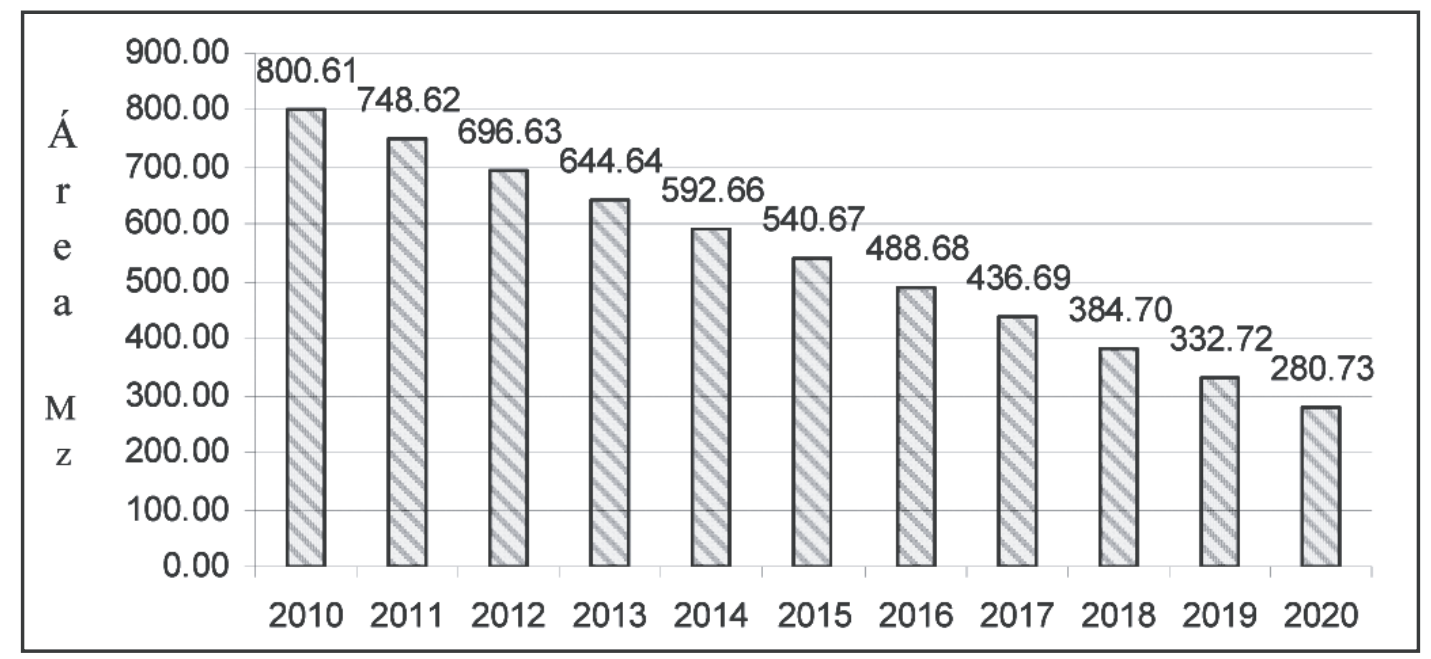

Figura 2. Proyección de sustitución de importaciones de papas en manzanas 2010-2020. Fuente: Adaptado de foasted (2005) y MAGFOR (2008).

Como se observa en la figura 2, la demanda proyectada es decreciente y es motivada por la disminución de las importaciones y por el aumento de la producción interna, y ésta a la vez es estimulada por lo atractivo del rubro en lo económico y social, al ser un alimento muy nutritivo y de importancia para la seguridad alimentaria nacional.
Coopamat, (2003) asegura que Nicaragua tiene capacidad para producir suficiente papa pero el problema radica en el alto costo y escasez de semilla para la producción. Hasta la fecha, se han logrado reproducir dos tipos de semilla de papa a partir de semilla sexual, Lila T y Papanica (INTA 2007).

Habiendo seleccionado ocho mercados de las cabeceras municipales del país, (Managua, Estelí, Jinotega, 
Tabla 2. Rendimientos promedios de papa en qq $\mathrm{Mz}^{-1}$ según país

\begin{tabular}{lccccccc}
\hline País & 2001 & 2002 & 2003 & 2004 & 2005 & 2006 & 2007 \\
\hline Costa Rica & 510.2 & 523.4 & 488.0 & 497.6 & 497.6 & 497.6 & 498.0 \\
El Salvador & 459.6 & 472.0 & 446.4 & 456.0 & 456.0 & 456.0 & 456.0 \\
Guatemala & 466.8 & 473.6 & 472.4 & 538.8 & 538.8 & 538.8 & 540.0 \\
Honduras & 340.6 & 331.0 & 362.0 & 345.4 & 323.8 & 323.8 & 340.0 \\
Nicaragua & 257.2 & 265.0 & 263.6 & 266.6 & 269.4 & 269.4 & 270.0
\end{tabular}

Fuente: Foasted (2005).

Matagalpa, León, Chinandega, Masaya y Granada) se estudió el tipo de demanda de la papa fresca, obteniendo los siguientes resultados.

La percepción por tipo de demanda del producto papa fresca se sitúa entre media y alta, y es proporcional al incremento poblacional, requiriéndose 385,187 quintales en el año, lo que representa el 36\% de la demanda nacional.

El segmento estudiado corresponde a consumidores de bajos ingresos que compra en los mercados municipales $y$, aunque la demanda es media, la tendencia es que aumente el consumo dado los múltiples usos del producto y atributos nutritivos del mismo.
Respecto a la procedencia, los encuestados opinaron que la mayor parte del producto que se comercializa en los mercados municipales es de origen nacional (79\%), en segundo lugar de Guatemala con un $16 \%$ y por último la procedente de Costa Rica, representando un 5\%.

Con relación a la percepción sobre los requisitos de calidad, los distribuidores opinaron que una papa de calidad debe reunir tres condiciones: estar sana, limpia y grande. La opinión sobre las características de la papa importada, esta enfocada a limpieza, buen tamaño y selección del producto. Entre los aspectos negativos se resalta la poca resistencia a la manipulación y sabor insípido del producto.

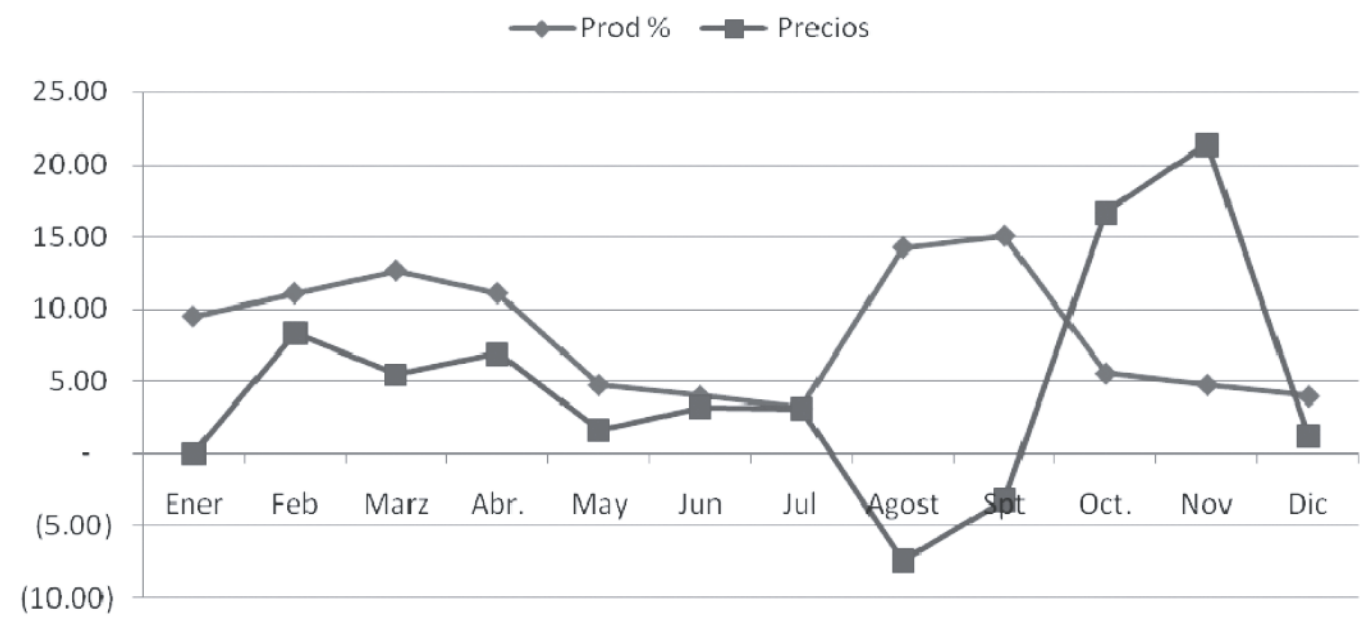

Figura 3. Ventana de mercado de acuerdo a los meses de mayor demanda.

La figura 3, refleja el comportamiento de los precios y la relación inversa con la producción a través de los doce meses del año. Como se observa las ventanas o meses con oportunidades de mercado del producto ocurre de mayo a julio, periodos donde la producción es menor y los precios son favorables. El otro momento sucede en el período de octubre a diciembre, donde los precios se incrementan y aumentan las importaciones de papa fresca para el consumo interno.
Sobre la percepción de las características de la papa nacional, se resalta la resistencia a la manipulación, el rendimiento y la duración o vida útil del producto. Entre los defectos mencionaron lo sucio y pequeño del producto.

La percepción de los distribuidores respecto a las características de la papa extranjera, resaltan la limpieza, el seleccionado y la diferenciación del producto que es suave y de buen tamaño. Con respecto a las limitaciones 


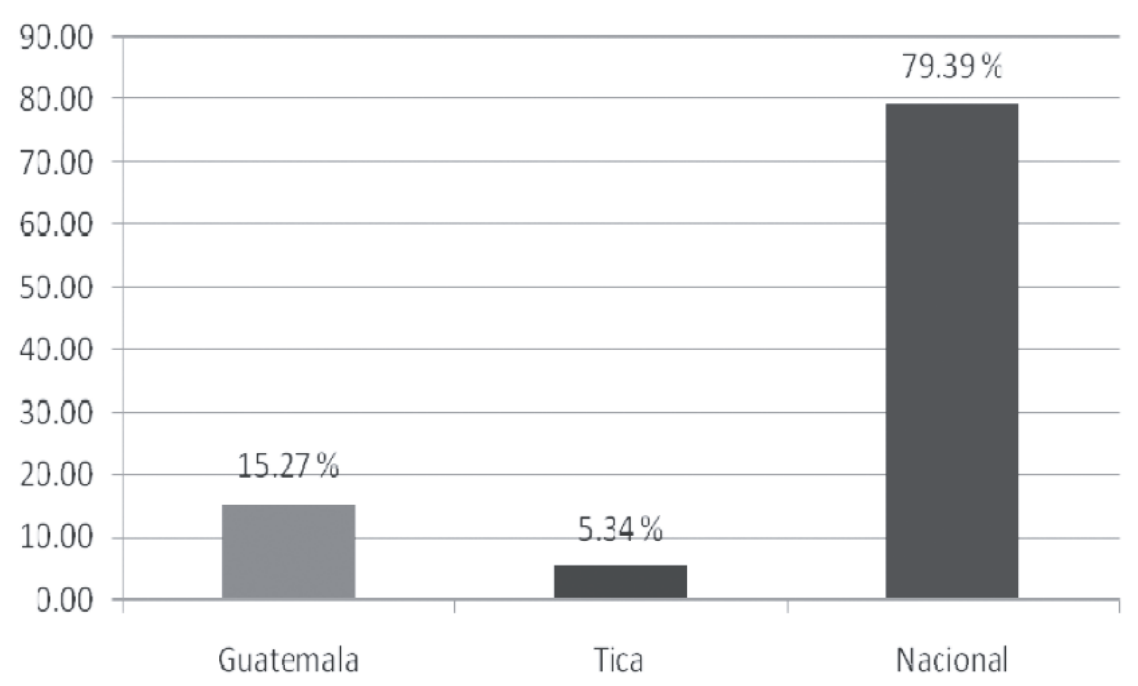

Figura 4. Procedencia de la papa y consumo nacional en porcentaje, 2007-2008. Fuente: Elaboración propia sobre la base de entrevistas con distribuidores.

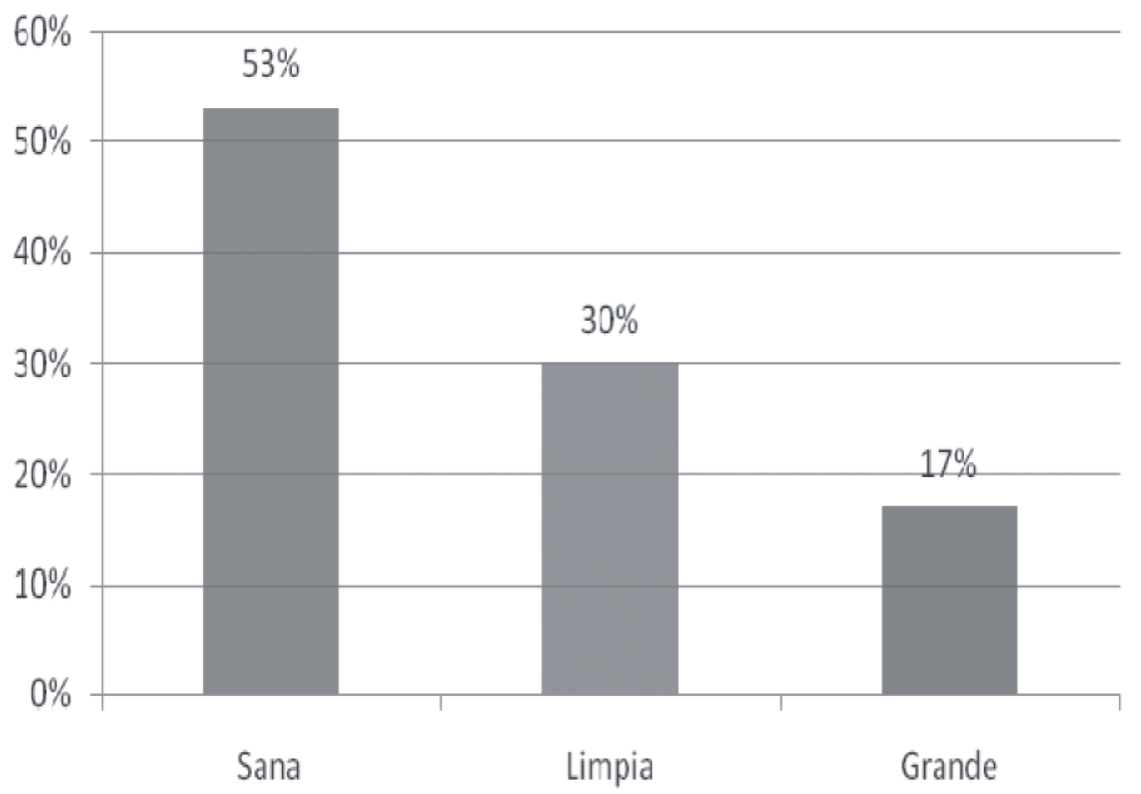

Figura 5. Percepción sobre los requisitos de calidad en los mercados municipales, 20072008. Fuente: Elaboración propia sobre la base de entrevistas con distribuidores.

expresan, que el producto es muy delicado o poco resistente y presenta un sabor insípido, por lo que el reto es producir una papa que reúna requisitos de resistencia, rendimiento, solidez como la nicaragüense y de buen tamaño como la importada.
Los diferentes puestos de distribución son abastecidos principalmente por mayoristas, en segundo lugar por el propio productor (Estelí, Jinotega, Matagalpa) y por último importadores y camioneros particulares. Cabe mencionar que la producción y distribución esta dominada por un reducido grupo de agentes económicos 


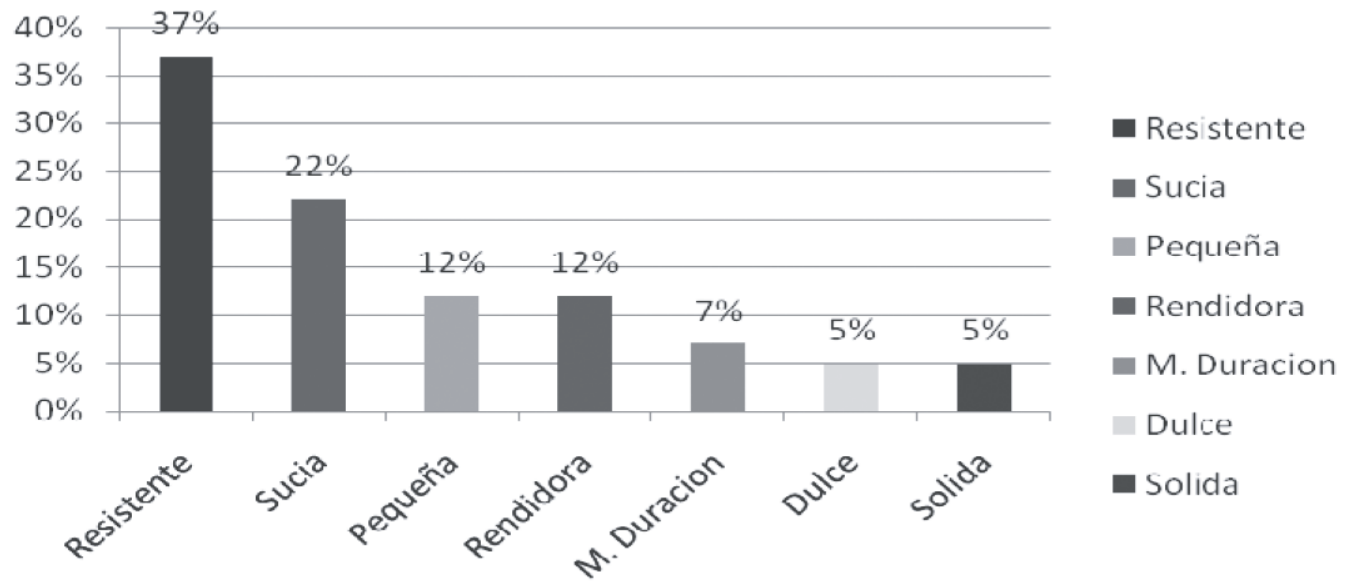

Figura 6. Percepción sobre las características de la papa nacional, 2007-2008. Fuente: Elaboración propia sobre la base de entrevistas con distribuidores.

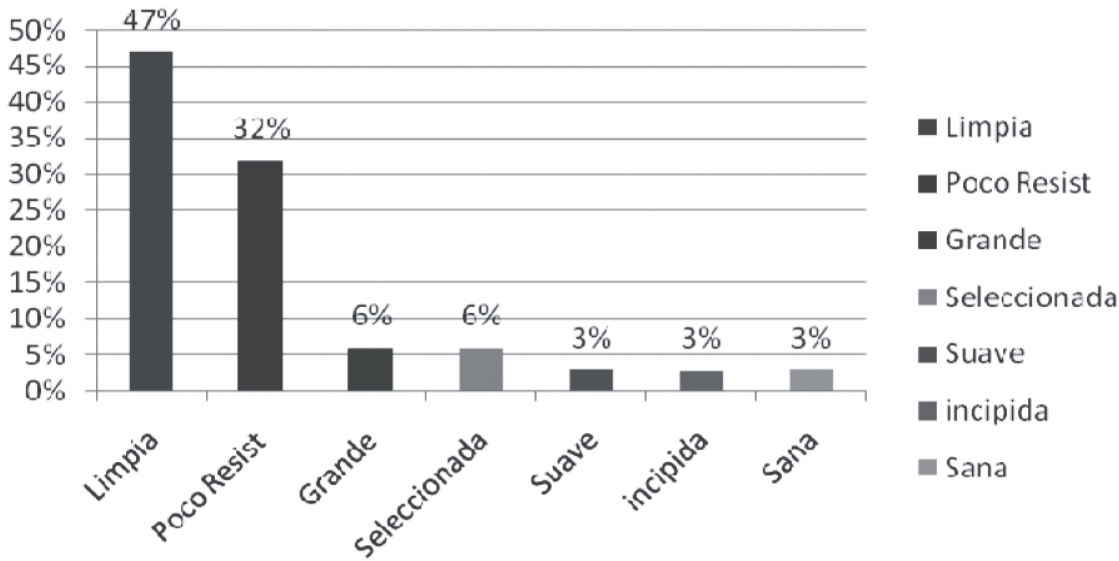

Figura 7. Percepción sobre las características de la papa importada, 2007-2008. Fuente: Elaboración propia sobre la base de entrevistas con distribuidores.

que disponen de los factores de producción, tecnología y mercado.

\section{CONCLUSIONES}

Existe potencial de mercado porque la demanda de papas es insatisfecha, importamos más del 40\%, existen áreas agrícolas aptas para el rubro (Estelí, Matagalpa, Jinotega), poca competencia en la producción interna, rubro atractivo como negocio por su aceptable rentabilidad y existen tendencias a incrementar el consumo percapita por su valor nutritivo e importancia en la seguridad alimentaria nacional.

Es un rubro atractivo como negocio por su viabilidad económica, comercialmente tiene demanda, se pueden muy nutritivo. Aunque la inversión es alta, el nivel tecnológico exigente y su contribución al medio ambiente no es muy significativa, la producción local es deficitaria y dependiente del exterior.

Para el futuro las tendencias orientan una reducción de las importaciones vía sustitución con producción nacional tanto para consumo fresco como de semilla de siembra, estimulado por la demanda del producto, rentabilidad atractiva y la importancia del rubro en la seguridad alimentaria nacional.

La producción de papas a nivel nacional registra los rendimientos más bajos con relación al resto de países de Centro América, debido a la baja calidad de la semilla, inadecuados métodos y técnicas de cultivo, alta incidencia de plagas y enfermedades y manejo post cosecha. 


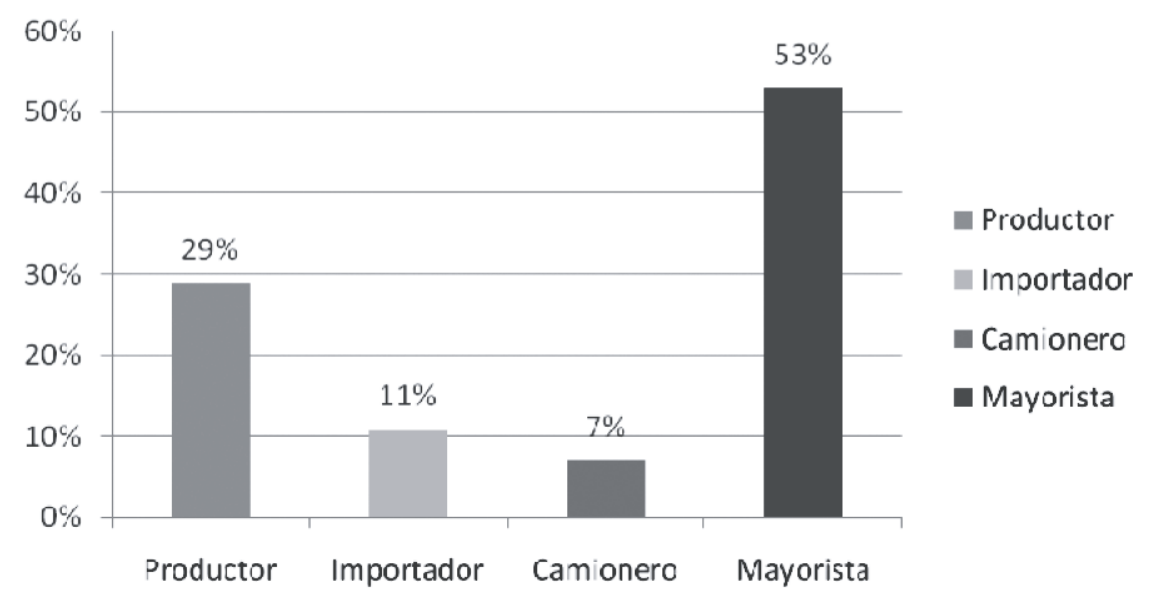

Figura 8. Percepción sobre el abastecimiento a los puestos de venta, 2007-2008.

Fuente: Elaboración propia sobre la base de entrevistas con distribuidores.

Las ventanas con oportunidades de mercado ocurren en los meses de mayo-julio y octubre a diciembre.

El segmento de distribuidores de los mercados municipales definió calidad como un producto que debe estar sano, limpio y grande.

Con base a las características de la papa nacional y extranjera; el reto es producir una variedad que reúna requisitos de resistencia, rendimiento, solides (рара Nica) y buen tamaño (para importada).

\section{RECOMENDACIONES}

Producir una semilla que reúna los requisitos de calidad como resistencia a plagas y enfermedades, al manejo postcosecha, buen rendimiento, solida y de buen tamaño.

Disponer de un banco de semillas certificadas disponibles según demanda y temporada.

Fortalecer las alianzas entre productores, Universidad Agraria e INTA para mejorar la calidad de la semilla para siembra.
Fortalecer los centros de investigación para el mejoramiento genético y la obtención de nuevas variedades adaptadas a nuestras condiciones y a los gustos y preferencias del consumidor.

Generar políticas de incentivos y estimulo hacia los productores para fomentar la producción nacional y reducir las importaciones del papas.

Fomentar el consumo de papas a través de ferias, comedores infantiles, bono productivo entre otros, dado su valor nutritivo para reforzar la política de seguridad alimentaria y nutricional.

Mayor asistencia técnica, capacitación y financiamiento a los productores para incrementar los rendimientos por unidad de área.

Aprovechar las ventanas de mercado o periodo de escasez del producto que ocurre en los meses de mayojulio y octubre a diciembre.

\section{REFERENCIAS BIBLIOGRÁFICA}

Ferre, J. 2003. Investigación de mercados estraticos. Editorial gestión 2000.

FOASTAD, 2005. Centro Agronegocios Tegucigalpa Honduras. santafeagro@amnettgu.com

INTA (Instituto Nicaragüense de Tecnología Agropecuario). 2007. Mercado de la semilla de papas.

MAGFOR (Ministerio Agropecuario y Forestal). 2008. Nicaragua logra reducir importaciones de papa en los tres

primeros meses del 2008. Divulgación y prensa.

MAGFOR (Ministerio agropecuario y forestal). 2008. Precios y mercados: Semana agropecuaria mes de agosto.

Méndez, C. 2005. Metodología: Guía para elaborar diseños de investigación. Editorial MacGraw Hill. 170 p.

Torrez, P. 2009. Comercialización de productos y servicios agrarios, primera edición 120 p. 